\title{
3D-analysis of plant microstructures: advantages and limitations of synchrotron X-ray microtomography
}

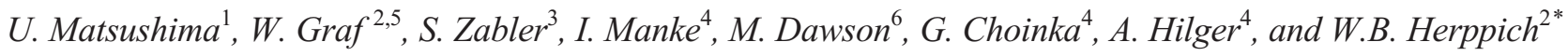 \\ ${ }^{1}$ Faculty of Agriculture, Iwate University, 3-18-8, Ueda, Morioka, Iwate, 020-8550, Japan \\ ${ }^{2}$ Department of Horticultural Engineering, Leibniz Institute for Agricultural Engineering Potsdam-Bornim, Max-Eyth-Allee 100, \\ 14469 Potsdam, Germany \\ ${ }^{3}$ Institute for Physics and Astronomy - Chair for X-ray microscopy, Julius-Maximillians Universitäty of Würzburg, \\ Campus Hubland Nord, Josef-Martin-Weg 63, 97074 Würzburg, Germany \\ ${ }^{4}$ SF3, Helmholtz Center Berlin for Materials and Energy, Hahn-Meitner-Platz 1, 14109 Berlin, Germany \\ ${ }^{5}$ Kuratorium für Technik und Bauwesen in der Landwirtschaft, Bartningstraße 49, 64289 Darmstadt, Germany \\ ${ }^{6}$ University of Salford, Newton Building 164, Salford, Greater Manchester, M5 4WT, UK
}

Received May 29, 2011; accepted October 15, 2011

\begin{abstract}
Synchrotron X-ray computer microtomography was used to analyze the microstructure of rose peduncles. Samples from three rose cultivars, differing in anatomy, were scanned to study the relation between tissue structure and peduncles mechanical strength. Additionally, chlorophyll fluorescence imaging and conventional light microscopy was applied to quantify possible irradiation-induced damage to plant physiology and tissue structure. The spatial resolution of synchrotron X-ray computer microtomography was sufficiently high to investigate the complex tissues of intact rose peduncles without the necessity of any preparation. However, synchrotron X-radiation induces two different types of damage on irradiated tissues. First, within a few hours after first X-ray exposure, there is a direct physical destruction of cell walls. In addition, a slow and delayed destruction of chlorophyll and, consequently, of photosynthetic activity occurred within hours/ days after the exposure. The results indicate that synchrotron X-ray computer microtomography is well suited for three-dimensional visualization of the microstructure of rose peduncles. However, in its current technique, synchrotron X-ray computer microtomography is not really non-destructive but induce tissue damage. Hence, this technique needs further optimization before it can be applied for time-series investigations of living plant materials.

$\mathrm{K}$ e y w o r d s: plant microstructure, chlorophyll fluorescence imaging, image analysis, mechanical strength, tissue damage
\end{abstract}

\section{INTRODUCTION}

Anatomy and histology of plants may change dramatically during development and senescence, but also in response to various environmental constraints, both at the macro-

*Corresponding author e-mail: wherppich@atb-potsdam.de scopic and at the microscopic scale (Graf, 2010). In addition to basic physiological research, comprehensive knowledge of the extent and the time course of changes in microstructures is essential to fully understand the respective underlying mechanisms. Thus, continuous and non-invasive analysis of plant microstructures is desirable. However, current high-resolution microscopy techniques eg SEM and TEM are inevitably destructive and discontinuous. On the other hand, the spatial resolution of conventional, non-destructive X-ray and magnetic resonance imaging (MRI) is too low to identify individual cells (Dhondt et al., 2010). In contrast, high-resolution tomography (Banhart et al., 2010), especially synchrotron X-ray computed microtomography $(\mathrm{SXC} \mu \mathrm{T})$ in combination with phase-contrast techniques may help to investigate the inner structure of plants at cellular resolutions in-situ (Cloetens et al., 2006; Mendoza et al., 2007).

In the early stages of SXC $\mu \mathrm{T}$ development, it was difficult to analyze soft matter such as plant tissues due to the low contrast in the X-ray images. Following the introduction of phase-contrast into X-ray tomography, it became possible to investigate such sensitive and complex material as a result of the much higher sensitivity of this technique to light elements (Bonse and Hart, 1965). The promising development of $\mathrm{SXC} \mu \mathrm{T}$ allows the close and continuous inspection of living tissue, even at the cellular level (Momose et al., 1996). 
Application of this method in plant science has only recently begun and studies have already demonstrated that synchrotron X-ray computer tompography is a promising technique with a high potential to investigate plant anatomy. It was successfully used to quantify the pore space within apple fruit tissues three-dimensionally (Mendoza et al., 2007) and to visualize the volume and structure of xylem net-works as well as the water-refilling process (Kim and Lee, 2010).

However, it is also clear that SXC $\mu \mathrm{T}$ is not yet optimized for plant samples in terms of radiation dose, spatial resolution and repeatability of measurements. Early investigations in macromolecular crystallography (Garman, 2010; Weik et al., 2000) established that synchrotron X-rays can damage and even destroy irradiated materials. The degree of this destruction is not negligible, but affects measurement results depending on radiation dose, dose rate and irradiation time (Cherezov et al., 2002). However, no systematic information on the effects of synchrotron X-radiation on living plant materials is available.

This study aimed to optimize the quality of SXC $\mu \mathrm{T}$ images of plant tissue by applying monochromatic X-radiation of various photon energies and to evaluate the ability of $\mathrm{SXC} \mu \mathrm{T}$ to continuously and non-invasively characterize the structural properties of sensitive living plant tissues.

\section{MATERIALS AND METHODS}

Roses (Rosa hybrida L.) of the cultivars Akito, Milva, and Red Giant were grown in a greenhouse at the Institute of Horticultural Sciences (Humboldt University Berlin, Germany). Heating temperature was set to $18^{\circ} \mathrm{C}$ while aeration temperature was $23^{\circ} \mathrm{C}$. Relative air humidity was in the range of 50 to $90 \%$ with large variations during the day. Natural light was supplemented with high pressure sodium lamps to yield a minimum photosynthetic photon flux density (PPFD) of $140 \mu \mathrm{mol} \mathrm{m} \mathrm{m}^{-2} \mathrm{~s}^{-1}$ for $18 \mathrm{~h}$. Plants were automatically trickle irrigated with nutrient solution in an open system. Applying the 'Japanese system' (bending down the blind or redundant stalks to increase leaf area for photosynthesis), plants were cultivated in 71 pots filled with commercial peat-coir-based substrate (Stender AG, Luckau, Germany) and placed in double rows (8.6 plants $\left.\mathrm{m}^{-2}\right)$ on tables. Roses were harvested at flower development stage 2 (Graf, 2010). After harvest, stems were placed in a Chrysal ${ }^{\circledR}$ professional solution (Prokon and Chrysal International B.V., Naarden, the Netherlands). Freshly harvested rose samples were transported to the experimental sites and stored in a refrigerator $\left(4^{\circ} \mathrm{C}\right)$ until the start of the experiments.

Synchrotron X-ray tomography of rose samples (Akito: 7 samples, Milva and Red Giant: 3 samples each) was conducted at the BAMline of the electron storage ring BESSY II at Helmholtz Center Berlin for Materials and Energy, Adlershof, Germany (Rack et al., 2008). To keep down radiation doses, in vitro CT scans of rose peduncles were taken with X-rays of 30, 40, $50 \mathrm{keV}$ photon energy. In this energy range, linear absorption is negligible for soft plant tissues. Hence, phase-contrast imaging was applied by positioning the detector $1.14 \mathrm{~m}$ away from the sample to reveal microscopic details (Cloetens et al., 1997).

At BAMline, the micro-CT setup is located $35 \mathrm{~m}$ downstream of the X-ray source. X-rays are monochromated by a double multilayer mirror yielding an energy window of $1-2 \%$. Rose samples were placed in a plastic funnel (outer diameter $8 \mathrm{~mm}$ ) glued on top of an acrylic glass vase (1 mm thick). To prevent the dehydration and heating of stem tissue during measurements, the vase was filled with water and connected to a reservoir by a silicone tube. Images were recorded by an indirect pixel detector (Rack et al., 2008) comprising a cadmium tungstate (CWO) scintillator screen and a Nikon lensfitted (1:18, f= $180 \mathrm{~mm}$; Nikon GmbH, Düsseldorf, Germany), back-illuminated, high-resolution, slow-scan CCD camera (VersArray:2048, Princeton Instruments, Trenton, USA). The field of view of the camera was $7.7 \times 7.7 \mathrm{~mm}$ yielding pixel size of $4.56 \mu \mathrm{m}$. The energy-dependent angular acceptance of the monochromator mirrors limited the effective image heights in the in vitro CT scans to $3.2,2.8$ and $2.4 \mathrm{~mm}$ (30, 40 and $50 \mathrm{keV}$, respectively).

For every 3D data set, 1500 unique projections were recorded covering 0 to $180^{\circ}$ rotation of the sample (incremental steps of $0.12^{\circ}$ ). The mean exposure time for each projection was $1.5 \mathrm{~s}$, hence, the mean total time of exposing samples to the X-ray radiation was $37.5 \mathrm{~min}$. After every 100 frames, a series of 10 flatfield images were recorded to correct for inhomogeneous beam profile and decreasing beam intensity. Images were Fourier-filtered to approximately reverse the Fresnel-propagation and to retrieve a twodimensional thickness projection.

The PyHST software package (European Synchrotron Research Facility, Mirone et al., 2010) was used to reconstruct a volume image from the phase-retrieved projections. The 3D voxel data were rendered using VGStudio (Volume Graphics GmbH, Heidelberg, Germany). Finally, horizontal and vertical CT images were processed using NI Vision Acquisition with LabView (National Instruments Co., Austin, USA) and ImageJ (Abramoff et al., 2004).

Simultaneously to SXC $\mu \mathrm{T}$ scans, chlorophyll fluorescence image analyses (CFI) were performed at $15 \mathrm{~min}$ intervals using a FluorCAM MF 740 system (PSI, Brno, Czech Republic). Controlled by WinXP compatible software (Fluor Cam 6), the system measures sequences of fluorescence images (CCD camera with F1.2/2.8-6 mm objective and shortpass filter, 12-bit resolution, 512 x 512 pixel; maximal frequency 50 images $\mathrm{s}^{-1}$ ) of objects (maximum field of view approx. $10 \times 13 \mathrm{~cm}$ ). Fluorescence is induced by two sets of 345 orange light emitting diodes $\left(\lambda_{\max }=620 \mathrm{~nm}\right)$ providing short (10-33 $\mu \mathrm{s}$ ), weak (approx. $5 \mu \mathrm{mol} \mathrm{m} \mathrm{s}^{-1}$ ) flashes as well as continuous actinic irradiation at a user-defined timing and irradiance. Short-term (1 s) saturation light pulses (max. $2500 \mu \mathrm{mol}$ photons $\mathrm{m}^{-2} \mathrm{~s}^{-1}$ ) were generated by a $250 \mathrm{~W}$ halogen lamp, equipped with an electronically-controlled shutter (Matsushima et al., 2009). 
Samples were dark-adapted for $10 \mathrm{~min}$ and then illuminated with the measuring light to induce the initial fluorescence, $F_{0}$ (von Willert et al., 1995). The maximum fluorescence signal, $F_{m}$, was elicited by a saturation pulse, which rapidly excites all chlorophyll molecules thus preventing any photochemical energy dissipation. The ratio $F_{v} / F_{m}\left(F_{v}=F_{m}-F_{0}=\right.$ variable fluorescence $)$ is an indicator of the potential maximum photochemical efficiency, and is thus a valuable tool in determining both capacity and stability of photosynthesis and its response to external constraints (von Willert et al., 1995).

In addition to these measurements, fresh, unstained, hand-cut slices of irradiated peduncle tissue were prepared at different times after the end of radiation. The samples were investigated by an optical microscope (DM RXA2, Leica Microsystems GmbH, Wetzlar, Germany) equipped with a Leica CCD camera.

\section{RESULTS AND DISCUSSION}

Spatial-resolution of the synchrotron X-ray tomography images was high enough to accurately investigate the complex microstructure of tissues such as vascular bundle elements and parenchyma cells in the pith of rose peduncles (Fig. 1). Cells and intercellular spaces were clearly discrimi- nated (Fig. 1A-h and A-v). Due to edge enhancement by phase-contrast imaging, the boundary lines ie the cell walls of each single cell were distinctly depicted. In addition, $\mathrm{SXC} \mu \mathrm{T}$ clearly discriminated between fully intact cells ie those containing cytoplasm, water-filled cells or xylem elements, and air-filled objects. The region of high X-ray attenuation in Fig. 1A-h (arrow 1) indicates an intact pith parenchyma cell, while dark areas represent empty, air-filled spaces. The thin cell walls, preserved after disappearance of the protoplasts, were distinctly visible in $\mathrm{SXC} \mu \mathrm{T}$ (Fig. 1A-h, arrow 2). In contrast to peduncles of both Red Giant and Akito roses, those of Milva (Fig. 1A, B, C) roses retained an intact pith and showed no air-filled cells in this tissue.

The effective irradiation dose to take one complete $\mathrm{SXC} \mu \mathrm{T}$ was smallest at a photon energy of $50 \mathrm{keV}$. As a result of the low photon flux, however, exposure time at this photon energy was more than double that at 30 and $40 \mathrm{keV}$ (Table 1). Hence, the time necessary to record single SXC $\mu \mathrm{T}$ images was $1.4,1.2$, and $3 \mathrm{~s}$ at photon energies of 30,40 , and $50 \mathrm{keV}$, respectively.

Furthermore, for one complete $\mathrm{SXC} \mu \mathrm{T}$, a total number of 1660 single images were taken, irrespective of the energy. Therefore, the time necessary to record an entire $\mathrm{SXC} \mu \mathrm{T}$ was longer at 30 than at $40 \mathrm{keV}$. If the longer overall recording
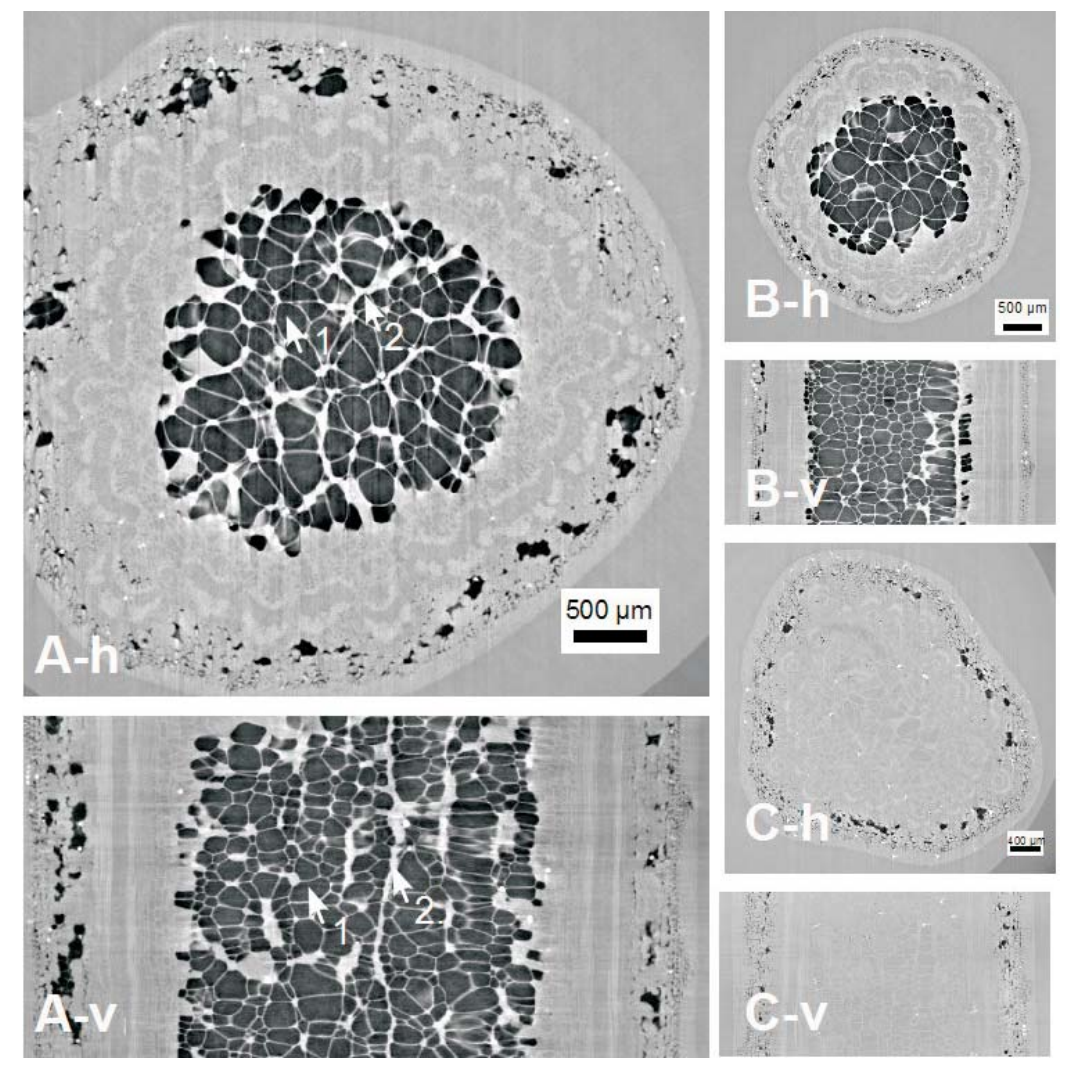

Fig. 1. Horizontal (-h) and vertical (-v) SXC $\mu$ T slices of Red Giant, Akito, and Milva (A, B, C) rose peduncles, taken at a photon energy of $40 \mathrm{keV}$. Thin walled, air-filled cells are indicated by low contrast dark areas (arrow 1); intact, cytoplasm-containing cells show a high $\mathrm{X}$-ray attenuation (arrow 2). 
time and the higher X-ray attenuation at $30 \mathrm{keV}$ are considered, the radiation effects of this photon energy on plant samples should be larger at this energy than at $40 \mathrm{keV}$. However, no significant difference between the quality of SXC $\mu \mathrm{T}$ image produced at 30 and $40 \mathrm{keV}$ could be established with the experimental setup used.

Overall, the quality of SXC $\mu \mathrm{T}$ images of Milva peduncles was not significantly affected by the photon energy used (Fig. 2). This also applied for Akito and Red Giant.

To evaluate the potential negative impact of synchrotron X-radiation on the physiological activity of the irradiated tissues, series of chlorophyll fluorescence images of rose peduncles were taken at regular intervals during and after recording SXC $\mu \mathrm{T}$. Figure 3A shows chlorophyll fluorescence images $\left(F_{v} / F_{m}\right)$ of a Red Giant rose peduncle 20, 80 and $615 \mathrm{~min}$ (A-2, A-3, A-4, respectively) after irradiation at $30 \mathrm{keV}$. At all given photon energies (data not explicitly shown), X-ray irradiation only very slowly and very locally affected photosynthesis. X-rays did not negatively affect maximum photochemical efficiency during (not shown) and immediately after irradiation. Within $2 \mathrm{~h}$ after the end of SXC $\mu \mathrm{T}$ measurements, $F_{m} / F_{v}$, and, hence, potential photo-

T a b l e 1. Conversion factor to effective dose and photon flux density of BAMline

\begin{tabular}{ccc}
\hline $\begin{array}{c}\text { Energy } \\
(\mathrm{keV})\end{array}$ & $\begin{array}{c}\text { Exposure time } \\
(\mathrm{s})\end{array}$ & $\begin{array}{c}\text { Photon flux density* } \\
\left(\text { photons s}^{-1} \mathrm{~mm}^{-2}\right)\end{array}$ \\
\hline 30 & 2324 & $7.2810^{10}$ \\
40 & 1992 & $4.9010^{10}$ \\
50 & 4980 & $1.1010^{10}$ \\
\hline
\end{tabular}

*(Rack et al., 2008): Photon flux density behind the double multilayer monochromator at the BAMline; measured with a ring energy of $1.7 \mathrm{GeV}$, a ring current of $200 \mathrm{~mA}$, a magnetic flux density of $7 \mathrm{~T}$ at the wavelength shifter insertion device and a distance from the source of $35 \mathrm{~m}$. At $7 \mathrm{keV}$, a flux density of $5.710^{10}$ photons s $\mathrm{sm}^{-1}$ was measured with a focused beam. Around $11 \mathrm{keV}$, typical flux reduction due to the tungsten $\mathrm{L}$ absorption edges can be seen (the multilayer coating consists of 150 double layers $\mathrm{W} / \mathrm{Si}$ ). synthetic activity did not change at all (Fig. 3B). Only after an additional $8 \mathrm{~h}$, did $F_{v} / F_{m}$ (solid squares in Fig. 3B) noticeably decrease in the irradiated region of the peduncle (profile along the dotted line in Fig. 3A-2). The low $F_{v} / F_{m}$ (black arrow in Fig. 3B) proves that photosynthesis was no longer active in the irradiated stem part. This response was valid for all rose cultivars investigated. Furthermore, photosynthetic activity declined to zero with very similar temporal dynamics at all X-ray energies tested.

After a delay of between 1 to $3 \mathrm{~h}$ and 1 day, the peduncle tissue pronouncedly weakened and became increasingly fragile, particularly samples of Milva which broke readily. In addition, irradiated peduncle regions started browning the change from green to brown taking several hours - and could be identified with the naked eye, irrespective of cultivar and X-ray photon energy. The optical microscopic images of hand-cut slices of the irradiated regions are presented for samples of all cultivars (Fig. 4). Browning was particularly evident in walls of cells of the hypodermis and of the vascular bundles. Again, these long-term reactions occurred independent of cultivar and X-ray photon energy.

Besides genetic and physiological control mechanisms, comprehensive knowledge of the anatomical and histological structure is indispensable to fully understand the function of plant organs or tissues and their dynamic changes during development and/or in response to environmental constraints.

In this context, high resolution $\mathrm{SXC} \mu \mathrm{T}$ is assumed to provide a very promising approach to non-invasively investigate plant microstructures (Mendoza et al., 2007). Even at the cellular level, this is valid without any need of additional preparations of samples (Cloetens et al., 2006; Dhondt et al., 2010).

The results presented here confirm the above statements. The resolution obtained with the applied system clearly allowed distinguishing the structural differences between air space and living cells in peduncle tissues of all investigated roses. For example, the content of pith parenchyma cells can be relatively easily visualized in 3-dimensional images. As indicated by the presented SXC $\mu \mathrm{Ts}$, only parenchyma cells of
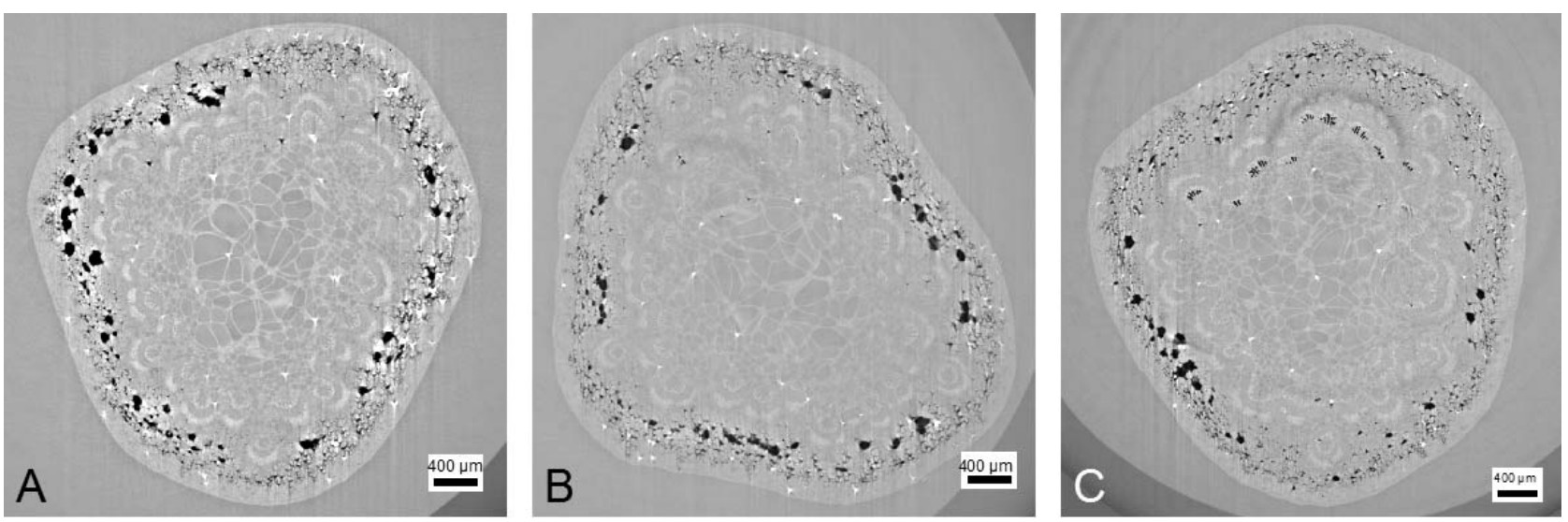

Fig. 2. Vertical slices of SXC $\mu \mathrm{T}$ images of Milva peduncles taken at 30, 40, and $50 \mathrm{keV}$ (A, B, C). 

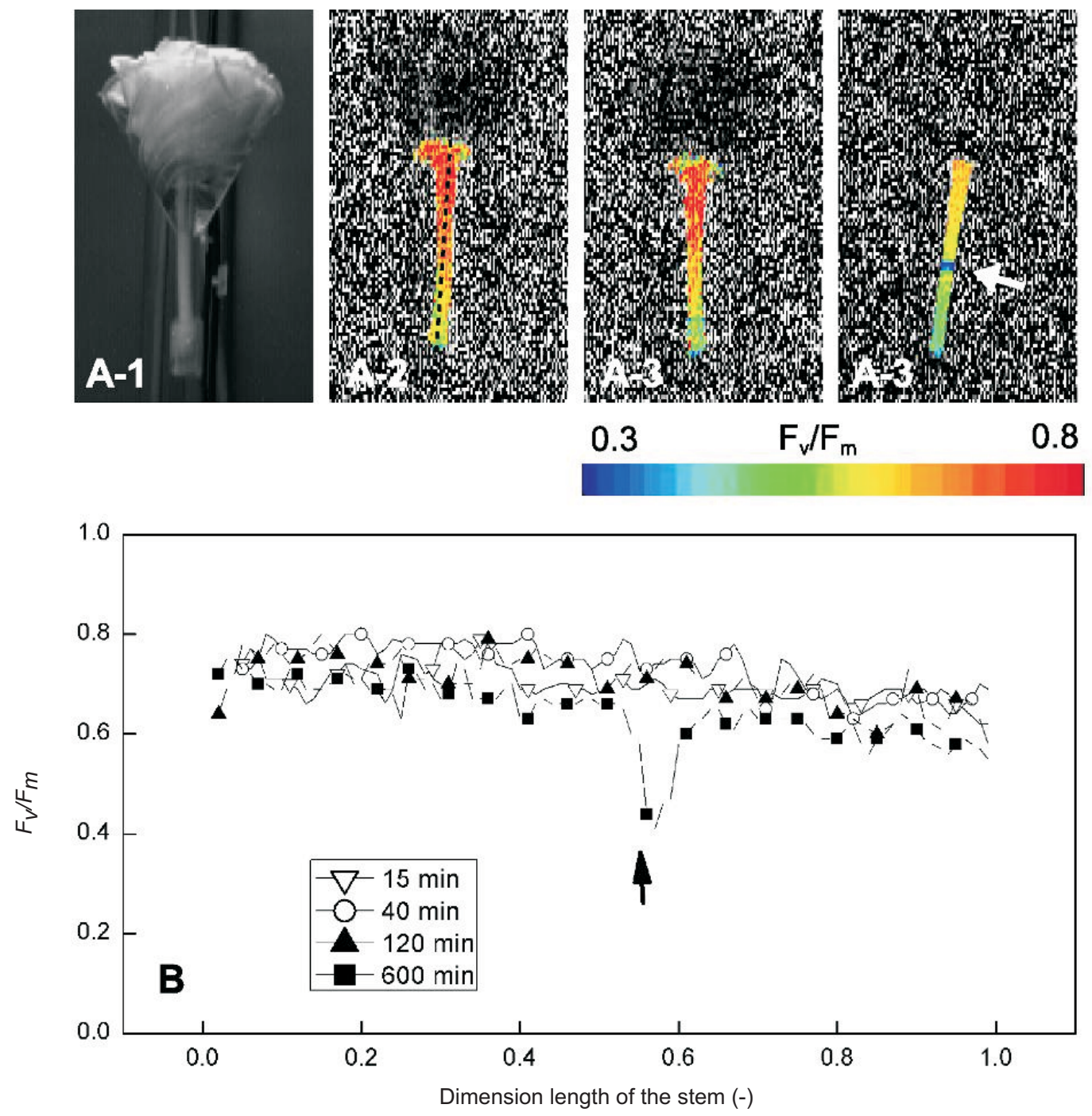

Fig. 3. Sample installation (A-1) and chlorophyll fluorescence images (maximum photochemical efficiency, $F_{v} / F_{m}$ ) of a Red Giant rose peduncle taken 20, 80, and $615 \mathrm{~min}$ (A-2, A-3, A-4) after irradiation at $30 \mathrm{keV}$ for $37.5 \mathrm{~min}$; B - photosynthetic activity profiles of chlorophyll fluorescence images taken at different times after synchrotron X-ray irradiation of a Red Giant peduncle at $30 \mathrm{keV}$.
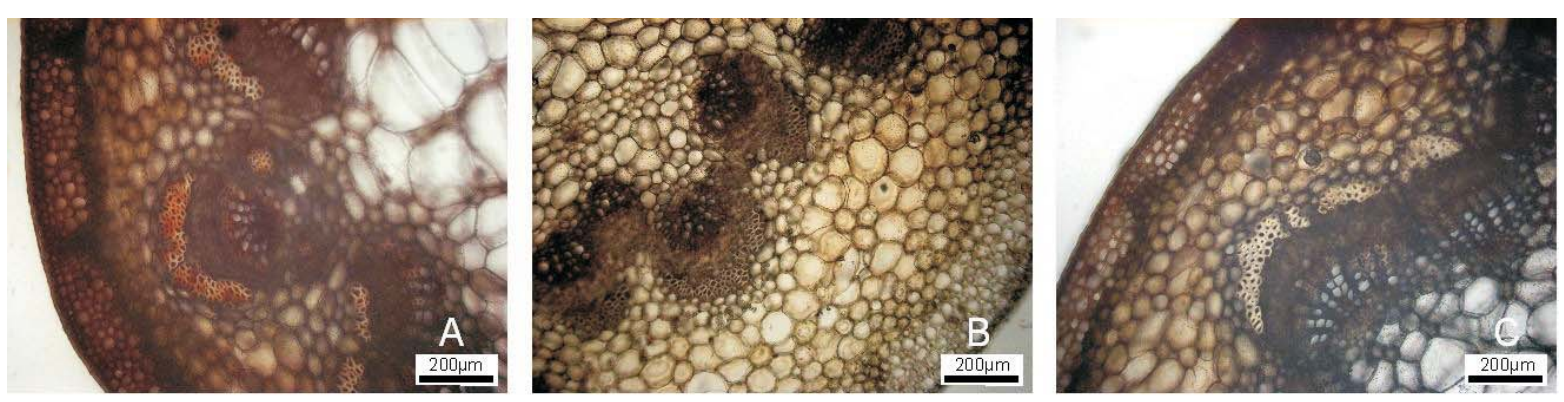

Fig. 4. Fresh cut slices of Red Giant, Akito, and Milva (A, B, C) rose peduncles irradiated at photon energies of $30 \mathrm{keV}$ for $37.5 \mathrm{~min}$. 
Milva roses still contained protoplasm. In contrast, pith cells of both Akito and Red Giant samples were mainly air filled. This pronounced structural difference may indicate differences in the developmental stage of rose peduncles. It has been assumed that this fact is directly related to stem mechanical properties and, hence, to the largely varying postharvest behaviour of the different cultivars (Graf, 2010).

Knowledge of the extent and the time course of possible changes of such functional plant microstructures is essential to understanding the underlying mechanisms. The presented data show that $\mathrm{SXC} \mu \mathrm{T}$ can, indeed, help to satisfy this requirement without direct interference with the product (Dhondt et al., 2010).

Synchrotron irradiation, on the other hand, is known to partially and specifically damage major components of plant cells, including proteins, lipids, pigments or cellulose (Cherezov et al., 2002; Simonis and Seuberling, 1973; Weik et al., 2000). In the presented experiments, impairment of functional tissue micro- and macrostructures due to synchrotron irradiation was obvious. Rapid changes in the physical structure of plant tissues may reduce $\mathrm{SXC} \mu \mathrm{T}$ quality.

In early studies (not shown), some rose peduncles broke immediately after irradiation. This harsh response might result from chemical changes in both plant cell membranes and cell walls. Intact biomembranes are vital for metabolic activity of plant cells. Free radicals, produced by X-ray irradiation, could trigger phase transformations in biomembranes leading to a loss of selective permeability and the ability to support gradients, resulting in loss of turgor and, ultimately, in cell death (Cherezov et al., 2002). Although peduncle cell turgor was not measured, turgor loss may have occurred but it seems not to be the major initial effect.

On the other hand, it is well-established that ionizing radiation rapidly induces destruction of cellulose in a doseeffect manner (Chunping et al., 2008). Irradiation decreases both crystallinity (Driscoll et al., 2009) and the degree of polymerization of this polysaccharide by molecule chain scission (Bouchard et al., 2006) and radical-induced or oxidative degradation. Such chemical reactions reduce the mechanical strength of cellulose (Bouchard et al., 2006). Because cellulose is a major component of cell walls that supports the structure and mechanical strength of tissues its destruction might be the main reason for the structural weakness of samples after synchrotron irradiation.

In fact, Milva peduncles were always the most fragile samples. Stems of this cultivar had the lowest content of structural carbohydrates (Graf, 2010). In addition, cell wall lignin content was also low in Milva roses compared to the other cultivars investigated (Graf, 2010). Lignin has been shown to inhibit the autoxidative degradation of cellulose (Schmidt et al., 1995) most probably due to its radical scavenging activity. In this context, it has been reported that, after irradiation, lignin-containing pulp retains its degree of polymerization and its tensile strength, but lignin-free softwood kraft pulp paper does not (Schmidt et al., 1995). This may explain the higher structural stability of Red Giant roses, which are known for their relatively high cell wall lignin content. In addition, the antioxidant properties of lignin may be responsible for the irradiation-induced discolourations in paper and pulp (Schmidt et al., 1995; Bouchard et al., 2006) and in rose peduncles, as observed in this investigation. However, there was no clear cultivar dependence of irradiation-induced browning.

Damage related to tissue structure and cell wall appeared to be relatively rapid events, occurring prior to any other disturbance of metabolic activity. On the other hand, decrease of photosynthetic activity, as indicated by the potential maximum efficiency of photosystem II $\left(F_{v} / F_{m}\right)$, only took place with a delay of several hours. This finding agrees well with earlier investigations on the effect of ionizing radiation on photosynthesis (Zill and Tolbert, 1958). In wheat leaves, $\mathrm{CO}_{2}$ fixation was much more sensitive to $\gamma$-irradiation than water splitting (Zill and Tolbert, 1958). Nevertheless, this decay was highly reversible within a few hours. In contrast, X-ray irradiation reduces the ability to synthesize chlorophyll by inhibiting the formation of protochlorophyillide (Price and Klein, 1962), while the conversion of synthesised protochlorophyll to chlorophyll was not inhibited. In addition, existing chlorophyll seems to be rather resistant to X-rays up to relative high doses (Simonis and Seuberling, 1973). Hence, the delayed reduction in $F_{v} / F_{m}$ may reflect the results of a 'normal' turn-over of chlorophyll at reduced or inhibited re-synthesis of this pigment.

Much of our knowledge on X-radiation damage has been gained from research of isolated lipid and biomembrane models (Cherezov et al., 2002) as well as from macromolecular crystallography (Garman, 2010). In such investigations, X-radiation freely and directly may interact with the irradiated material. Synchrotron radiation leads to both primary interactions between photons and the moieties that suffer specific damage, and secondary reactions, mediated by free radicals generated by the X-ray photons either within the protein or within the matrix (Weik et al., 2000). In addition, X-radiation interacts with water in and around the target. This results in a cascade of reactions giving rise to reactive oxygen species (ROS), hydroxyl radicals, hydrated electrons and hydrogen atoms, the relative amount of which depend on temperature, $\mathrm{pH}$ and other factors (Garman, 2010). However, in a living plant system, radical scavengers are readily available largely reducing the risk of the direct or indirect effect of free radicals on physiological activities such as photosynthesis.

Hence, the delay in $\mathrm{F}_{\mathrm{V}} / \mathrm{F}_{\mathrm{m}}$ reduction may indicate that $\mathrm{X}$-radiation did not directly destroy photosynthetic protein complexes or photosynthetic pigments. In contrast, radicals may have simultaneously damaged many steps of the entire metabolic pathway. Gradually, the function of the entire pathway declined. Such long-lived radical-mediated aftereffects have also been reported in the degradation of cellulose (Chunping et al., 2008; Driscoll et al., 2009). 
In the case of conventional X-ray irradiation, damage mainly comes from high X-ray doses. However, for synchrotron X-radiation, the effects of beam flux and energy must also be considered. In this context, beam heating at higher dose rates may have resulted in a pronounced local temperature increase (Cherezov et al., 2002). For X-ray crystallographic investigations of microstructures such as proteins, cooling techniques are often employed to reduce radiation damage. The radical species produced by the energy loss of the beam would diffuse much slower, or even not at all, and the speed of the chain reactions will be reduced. This could effectively reduce the collateral damage (Garman, 2010). However, it is difficult to sufficiently cool living intact plant materials during irradiation because temperatures should not fall below freezing point.

In addition, the direct effects of radiation energy should also be taken into account. According to Cherezov et al. (2002), there is no major damaging effect of beam energies between 9 and $17 \mathrm{keV}$. However, in the presented experiments, the X-ray photon energies applied ranged from 30 to $50 \mathrm{keV}$, which inevitably resulted in higher effective radiation doses compared to the study of Cherezov et al. (2002). However, at higher energies X-ray photons are less easy absorbed by tissue components.

To create high resolution $\mathrm{SXC} \mu \mathrm{T}$ images, a certain number of photons is necessary. Similar to the effective dose, the total number of photons is the product of photon flux density and exposure time. Even though the differences were only marginal, image quality and resolution seemed to be highest at $50 \mathrm{keV}$ in this study and both the effective dose and the number of photons were smallest at this photon energy. Therefore, to improve image quality and resolution at the lower X-ray energies of 40 and $30 \mathrm{keV}$, it may be necessary to reduce the number of photons at those levels. This would, however, unnecessarily prolong total exposure time and increase the probability of tissue destruction, thus reversing the positive effects.

To employ SXC $\mu \mathrm{T}$ for non-destructive, continuous investigations of plant microanatomy, further optimization concerning dose and image quality is needed. In medical CT and radiology, X-ray imaging techniques have to achieve the maximum image quality with the lowest possible dose, $e g$ by limiting the number of projections. Possible solutions may be to use beam shutters to protect samples from unnecessary exposure during readout or to artificially slow down scans in order to allow the tissues to, at least partially, self-repair ie regain chemical equilibrium after ionization. In summary, raising the energy of the beam and employing phase-retrieval was one important step to dose reduction, but surely not the last.

\section{CONCLUSIONS}

1. The inner structure of intact rose peduncles was successfully investigated using synchrotron X-ray imaging. With its high resolution, $\mathrm{SXC} \mu \mathrm{T}$ is well applicable for the non-destructive three-dimensional imaging of plant material. Hence, this technique can help to clarify the involvement of distinct structural differences in vascular bundle and peduncle pith structure in the mechanisms determining the different vase life of the rose cultivars studied.

2. During continuous investigation of intact plant samples by $\mathrm{SXC} \mu \mathrm{T}$, it is necessary to take into account the potential damaging effects of the irradiation. Synchrotron X-radiation seems to induce two different types of damage on irradiated plant samples. Within a few hours there is a very rapid and direct physical destruction of cell walls. In addition, a slow and delayed destruction of the tissue occurs, which may be of related to direct effects on metabolic pathways such as photosynthesis.

3. $\mathrm{SXC} \mu \mathrm{T}$ is a valuable tool for the non-invasive analysis of plant microstructures. Due to the high photon fluxes and energy of the synchrotron X-ray beams, however, that are currently still necessary for high-resolution imaging, plant tissues may be severely damaged during CT scans. This may limit the use of this method in continuous investigation.

\section{REFERENCES}

Abramoff M.D., Magelhaes P.J., and Ram S.J., 2004. Image processing with ImageJ. Biophotonics Int., 11, 36-42.

Banhart J., Borbély A., Dzieciol M., Manke I., Kardjilov N., Kaysser-Pyzalla A.R., Garcia-Moreno F., Strobl M., and Treimer W., 2010. X-ray and neutron imaging - complementary techniques for materials science and engineering. Int. J. Mat. Res., 9, 1069-1079.

Bonse U. and Hart M., 1965. An X-ray interferometer. Appl. Phys. Lett., 6, 155-156.

Bouchard J., Méthot M., and Jordan B., 2006. The effects of ionizing radiation on the cellulose of woodfree paper. Cellulose, 13, 601-610.

Cherezov V., Riedl K.M., and Caffrey M., 2002. Too hot to handle? Synchrotron X-ray damage of lipid membranes and mesophases. J. Synchrotron Radiat., 9, 333-341.

Chunping Y., Zhiqiang S., Gucose Y., and Jianling W., 2008. Effect and aftereffect of $\gamma$ radiation pretreatment on enzymatic hydrolysis of wheat straw. Biores. Technol., 99, 6240-6245.

Cloetens P., Mache R., Schlenker M., and Lerbs-Mache S., 2006. Quantitative phase tomography of Arabidopsis seeds reveals intercellular void network. Proc. Nat. Acad. Sci. USA, 103, 14626-14630.

Cloetens P., Pateyron-Salomé M., Buffière J., Peix G., Baruchel J., Peyrin F., and Schlenker M., 1997. Observation of microstructure and damage in materials by phase sensitive radiography and tomography. J. Appl. Phys., 81, 5878-5886.

Dhondt S., Vanhaeren H., van Loo D., Cnudde V., and Inzé D., 2010. Plant structure visualization by high-resolution $X$-ray computed tomography. Trends Plant Sci., 15, 419-433.

Driscoll M., Stipanovic A., Winter W., Cheng K., Manning M., Spiese J., Galloway R.A., and Cleland M.R., 2009. Electron beam irradiation of cellulose. Rad. Phys. Chem., $78,539-542$. 
Garman E.F., 2010. Radiation damage in macromolecular crystallography: what is it and why should we care? Acta Cryst., 66, 339-351.

Graf W., 2010. Einfluss von Funktion und Struktur des Blütenstiels auf die Nacherntephase von Schnittrosen. Dissertation.de - Verlag im Internet GmbH, Berlin, Germany.

Kim H.K. and Lee S.J., 2010. Synchrotron X-ray imaging for nondestructive monitoring of sap flow dynamics through xylem vessel elements in rice leaves. New Phytol., 188, 1085-1098.

Matsushima U., Kardjilov N., Hilger A., Manke I., Shono H., and Herppich W.B., 2009. Visualization of water usage and photosynthetic activity of street trees exposed to $2 \mathrm{ppm}$ of $\mathrm{SO}_{2}-\mathrm{a}$ combined evaluation by cold neutron and chlorophyll fluorescence imaging. Nucl. Instrum. Meth. A, 605, 185-187.

Mendoza F., Verboven P., Mebatsion H.K., Kerckhofs G., Wevers M., and Nicolaï B., 2007. Three-dimensional pore space quantification of apple tissue using X-ray computed microtomography. Planta, 226, 559-570.

Mirone A., Wilcke R., Hammersley A., and Ferrero C., 2010. PyHST - High Speed Tomographic Reconstruction, http:// www.esrf.eu/UsersAndScience/Experiments/TBS/SciSoft.

Momose A., Takeda T., Itai Y., and Hirano K., 1996. Phasecontrast X-ray computed tomography for observing biological soft tissues. Nature Med., 2, 473-475.
Price L. and Klein W.H., 1962. Chlorophyll synthesis in X-irradiated etiolated bean leaf tissue. Rad. Bot., 1, 269-275.

Rack A., Zabler S., Müller B.R., Riesemeier H., Weidemann G., Lange A., Goebbels J., Hentschel M., and Görner W., 2008. High resolution synchrotron-based radiography and tomography using hard X-rays at the BAMline (Bessy II). Nucl. Instrum. Meth. A, 586, 327-344.

Schmidt J.A., Rye C.S., and Gurmagui N., 1995. Lignin inhibits autoxidative degradation of cellulose. Polymer Degrad. Stabil., 49, 291-297.

Simonis W. and Seuberling H.B., 1973. Sensitivity of chlorophyll formation and of increase of NADP-dependent GDP-activity in greening Euglena gracilis to X-ray and other inhibitors of protein synthesis. Rad. Bot., 13, 297-300.

von Willert D.J., Matyssek R., and Herppich W.B., 1995. Experimentelle Pflanzenökologie: Grundlagen und Anwendungen. Georg Thieme Verlag, Stuttgart, Germany.

Weik M., Ravelli R.G., Kryger G., McSweeney S., Raves M.L., Harel M., Gros P., Silman I., Kroon J., and Sussman J.L., 2000. Specific chemical and structural damage to proteins produced by synchrotron radiation. Proc. Nat. Acad. Sci., USA, 97, 623-628.

Zill L.P. and Tolbert N.E., 1958. The effects of ionizing and ultraviolet radiations on photosynthesis. Arch. Biochem. Biophys., 76, 196-203. 